\title{
DESIGN AND TECHNIQUE OF OPERATION OF A DOUBLE CRYSTAL SPECTROMETER
}

\author{
By Jesse W. M. Dumond and Archer Hoyt \\ California Institute of Technology, Pasadena
}

(Received November 8,1930 )

Abstract

The double crystal $\mathrm{x}$-ray spectrometer is distinctly different in principle from the single crystal spectrometer and calls therefore for a completely new design rather than an adaptation of single spectrometer design. One such new design is described in this paper. Emphasis is laid on the fact that the wave-length selected by the process of two successive crystal reflections in any prescribed orders depends solely on the dihedral angle between the crystals. The advantages of rotating both crystals in equal and opposite directions with respect to a plane through their axes of rotation are pointed out and a spectrometer designed to accomplish this is described. In this spectrometer the turning of a single shaft drives the two crystals, the spectrometer as a whole and the $\mathrm{x}$-ray tube, at the proper angular rates about the proper centers to insure that the $\mathrm{x}$-ray beam will at all times remain centered on the crystal faces and the window of the stationary ion chamber. It is pointed out that spurious fluctuations may be introduced in spectral curves by the $x$-ray beam migrating across small steps in the cleavage surface reflecting the radiation. Description of design covers design of spectrometer and turning mechanisms, the tube housing, lead shields, the detecting system consisting of an ion chamber with internal grid of special design to minimize natural leak and a Hoffmann vacuum electrometer connected to it through a short evacuated shield. Description of operating technique covers optical method of accurately orienting the crystal faces, methods of aligning the tube, precautions to eliminate background.

$T^{\prime}$ HE Double crystal $x$-ray spectrometer first used in this country by A. $\mathrm{H}$. Compton was developed by Bergen Davis ${ }^{1}$ and his collaborators and has been very thoroughly investigated on the theoretical side by Schwarzschild ${ }^{2}$ and on the experimental side by S. K. Allison ${ }^{3}$ and others. ${ }^{4}$ Its field of applicability seems to fall into two classes. First it may be used to study the properties of the crystals themselves (reflecting power, lattice perfections, etc. ${ }^{5}$ ) Secondly it may be used as an $\mathrm{x}$-ray spectrometer for exploring spectra (satellites, fine structure line breadths, Compton effect, etc.) ${ }^{6}$ In the development stage it was natural not to differentiate sharply in the design of double spectrometers for these different classes of work. It was also perfectly natural to adapt the general features of single crystal spectrometer design to the (1928)

${ }^{1}$ Bergen Davis, Phys. Rev. 17, 608 (1921), Phys. Rev. 27, 18 (1926), Phys. Rev. 32, 331

${ }^{2}$ Schwarzschild, Phys. Rev. 32, 162 (1928).

3 Allison, Phys. Rev. 34, 176 (1929), 35, 1476 (1930).

4 Ehrenberg and Mark, Zeits. f. Physik 42, 807 (1927).

- Davis \& Purks, Phys. Rev. 34, 181 (1929).

6 DuMond \& Hoyt, Phys. Rev. 36, 799 (1930), Bearden, Phys. Rev. 36, 791 (1930), Richtmyer \& Taylor, Phys. Rev. 36, 1044, (1930). 
double spectrometer. The authors feel however that now that so much is known about the possibilities and applications of this instrument the time has come to design it on its own merits in such a way as best to accomplish some particular function.

The double spectrometer here described was developed for the second of the above mentioned uses - the study of $\mathrm{x}$-ray spectra. It is designed to be used entirely in the antiparallel positions $(n, n)$ (Allison's notation) ${ }^{7}$ though slight modifications would permit "straddling two different orders."

The authors feel that much of the apparent complexity arising in connection with the double spectrometer comes from the introduction of such artificial frames of reference as the axis of rotation of the pivots supporting the crystals, or the axis of the slits which latter in the double spectrometer play an entirely different role from the slits of a single spectrometer.

The Important Thing is The Dihedral Angle Between The Crystals

It is of cardinal importance to note just what is measured in using the double spectrometer. A beam of $\mathrm{x}$-rays reflected from a cleavage face of a

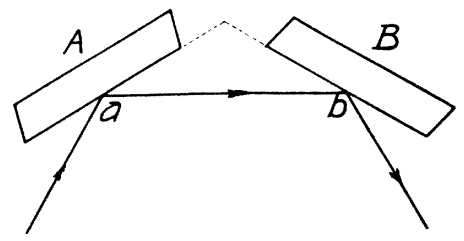

Fig. 1. Symmetrical ray selected by the bicrystalline reflection.

calcite crystal falls upon the face of a second calcite crystal and is again reflected into an ionization chamber. One or both of these crystals is turned slightly about an axis in the plane of the reflecting face and the ionization currents in the ion chamber are plotted as a function of the rocking angles of the crystal. For any given angle setting the two successive calcite reflections select out of the entire $x$-ray spectrum an exceedingly sharply defined narrow region of wave-lengths.

Precisely what angle determines the wave-length selected by the double $\mathrm{x}$-ray reflection? Is it the angle through which one crystal rotates with respect to the slit system? Obviously not, since the horizontal slit width has nothing to do with the resolution of the double spectrometer. Neither do the pivots about which the crystals rotate constitute in any sense the system of reference with respect to which the rotation must be measured which determines the selected wave-length. The authors wish to emphasize most strongly that the important thing is the dihedral angle formed by the two crystal faces themselves. Let us consider for simplicity the case of first order reflection from two similar calcites in the anti-parallel position $(1,1)$. Fig. 1 represents the dihedral angle formed by the two crystals. Obviously the only rays which can be reflected twice selectively in the first order by both crystals are the

' Allison \& Williams, Phys. Rev. 35, 149 (1930). 
symmetrical rays which make the same glancing angle at crystal $A$ as at crystal $B$. A symmetrical ray, $a b$, is shown in Fig. 1 passing from crystal $A$ to crystal $B$.

Define a plane normal to the dihedral angle formed by the crystal faces which for brevity and in accord with other writers we will call the "horizontal plane," (plane of the paper in Fig. 1). Now the ray, $a, b$, need not be parallel to the "horizontal plane" in order that it shall make equal glancing angles at each crystal and therefore be reflected. Such a ray oblique to the horizontal plane will in accord with Davis' terminology be said to have an angle of vertical divergence namely the angle $\alpha$ of obliquity with the horizontal plane. The projection of such an oblique ray will in Fig. 1 still be represented by the line, $a, b$, but the ray will in space actually suffer reflection at both crystals under a smaller glancing angle than rays which are not oblique. See Fig. 2.

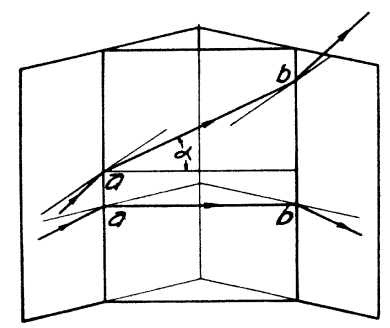

Fig. 2. Horizontal and oblique symmetrical rays.

We can assert then that the glancing angle (Bragg angle) selected by the process of the double selective reflection is half of the supplement of the dihedral angle between the crystal faces for horizontal rays and less than this for oblique rays. The band of wave-lengths selected then by the double reflection has a sharp limit on the long wave-length side determined only by the dihedral angle between the crystals. The band selected can be made as narrow as one pleases by limiting the vertical divergence.

Nothing in the above argument is made to depend on or refer to the pivots or axes of rotation of the crystals. It is of course obviously desirable that these axes of rotation should be parallel to the edge of the dihedral angle formed by the two crystal faces in order readily to permit the measurement of that dihedral angle and also to maintain the "horizontal ray" in a permanently fixed position with respect to the frame of the apparatus as the crystals rotate. The pivots, however, have nothing at all to do with the selective action of the double reflection and the dihedral angle between the crystals has everything to do with it. If this very fundamental fact is remembered all uncertainty as to the effect of various errors of alignment, etc., will vanish.

\section{Band Pass Filter Width}

The theoretical spectral intensity curve of the wave-length band selected from a uniform or "flat" spectral energy distribution by the double crystal reflection in the $(n, n)$ position with perfect crystals is shown in Fig. 3. It is 
bounded on the long wave side by a vertical discontinuity and falls off on the short wave side more gradually, meeting the axis of abscissae so as to have a base breadth $\Delta \lambda$ related to the maximum vertical divergence permitted by the system of lead stops limiting the x-ray beam. Referring to Fig. 4 let $\theta_{0}$

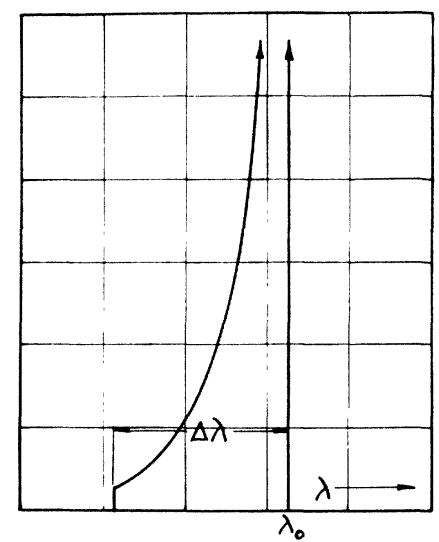

Fig. 3. Spectral intensity distribution selected by geometry of bicrystalline reflection with crystals fixed. This curve is not to be confused with the shape of a spectral line obtained by plotting reflected $x$-ray intensity against the dihedral angle between the crystals (or half of its supplement). Such line shapes are due to the overlapping of the "natural" line with the above curve as the latter is displaced spectrally with rotation of the crystals. The equation of the above curve is $y=k /\left[2\left(\lambda_{0}-\lambda_{\alpha}\right) \lambda_{0}\right]^{1 / 2} ; \lambda_{0}\left(1-\frac{1}{2} \alpha^{2}\right)<\lambda_{\alpha}<\lambda_{0}$ calculated on the assumption that $\mathrm{x}$-ray intensity is uniformly distributed over the permitted range of vertical divergences. The curve has a finite area in spite of the infinite ordinate at $\lambda_{0}$. In a spectral exploration as such a curve progressively overlaps a spectral line the intensity will therefore always remain finite.

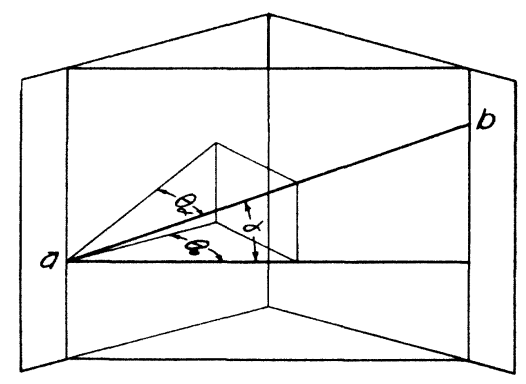

Fig. 4. Dependence of glancing angle on vertical divergence.

be the Bragg angle for a horizontally reflected ray, $\theta_{\alpha}$ the Bragg angle for a ray of vertical divergence $\alpha$. Then from the geometry of the case

and since

$$
\sin \theta_{\alpha}=\sin \theta_{0} \cos \alpha
$$

$$
n \lambda / 2 d=\sin \theta
$$

we have

$$
\begin{aligned}
\lambda_{\alpha} & =\lambda_{0} \cos \alpha \\
\Delta \lambda & =\lambda_{0}-\lambda_{\alpha}=\lambda_{0}(1-\cos \alpha)
\end{aligned}
$$


and since in practice $\alpha$ is restricted to small values we have as a good approximation

$$
\Delta \lambda=\frac{1}{2} \lambda_{0} \alpha^{2} .
$$

\section{Distortion of Lines by Vertical Divergences}

The characteristic curve of Fig. 3 which may be regarded as the "frequency response curve" of the spectrograph for any given setting of the crystals displaces itself bodily toward longer wave-lengths as the dihedral angle between the crystal faces closes up. In this exploring process if a sharp line is encountered at some particular wave-length the ionization chamber will respond abruptly on the short wave side of the line and will diminish more gradually as the characteristic exploring curve moves to the long wave side of the lines. For this reason the distortion of spectral lines by the geometrical effect of vertical divergence alone is the reverse of Fig. 3, the discontinuous edge being on the short wave side. A good example of such distortions appears in Fig. 11 reproduced from a previous article ${ }^{8}$ published by the authors. It is an easy matter however to so limit the vertical divergence that its effect in broadening spectral lines is negligble in comparison to the effects of crystal imperfections and natural $x$-ray line breadths combined.

\section{Objections to Rocking Only One Crystal}

The fact that the rays selected by the mechanism of the double reflection are symmetrically orientated with respect to the dihedral angle between the crystal faces immediately suggests that in exploring the spectrum both crystals should be simultaneously rotated in equal and opposite directions with respect to a plane through their axes of rotation.

As a matter of fact however most double spectrometers are built so that only one crystal is rotated in exploring the spectrum. This necessitates rotating the other crystal from time to time and also changing the relative position of the two crystals to prevent the beam from falling partly or wholly off the reflecting faces. As one crystal alone is rotated the selected beam must of necessity progress across the faces of the two crystals in order that it shall continue to make equal glancing angles with both crystal faces. The objections to this procedure are:

1. The crystals may present different reflecting powers in different parts of their surfaces so that spurious intensity fluctuations would be introduced in a spectral exploration. In particular all calcite cleavage faces have multitudinous small "steps" where the fracture changes abruptly from one plane to another one parallel to it. At glancing angles such steps obviously cast shadows in the reflected ray which will diminish its intensity in different degrees according to whether the beam falls on a part of the surface with high steps or a part in which steps are nearly absent. If the beam is obliged to migrate across the crystal face these steps will surely introduce undesirable spurious fluctuations. Even though the beam remains centered at one point of the crystal surface the area of crystal surface employed in reflection will

${ }^{8}$ DuMond and Hoyt, Phys. Rev. 36, 799 (1930). 
change with the glancing angle and some spurious fluctuations may thus be introduced by cleavage steps. These must however be regarded as an irreducible minimum.

2. If the source of radiation is not a point but has "horizontal" extension so as to permit reflection of one wave-length over a considerable area of the crystal a part of the beam which would otherwise be reflected may fall off one edge of the crystal introducing again spurious intensity fluctuations in a spectral exploration.

3. To avoid the above danger either very long crystals or a much reduced reflecting area must be used, both of which are rather serious difficulties.

4. The initial task of aligning the double spectrometer and locating a given spectral line is much harder in the instruments whose crystals do not rotate simultaneously.

As a corollary to what has just been said it is evidently desirable also to maintain the focal spot of the x-ray tube or other radiation source as well as the ionization chamber window always so situated with respect to the spectrometer that on one hand the selected beam from the tube will remain centered on the same reflecting region of the crystal faces for all glancing angles and on the other hand the selected radiation will always strike the ion chamber window at the same point. It is easy to see that large spurious fluctuations could be introduced by poor alignment of the beam entering a narrow ion chamber window and even with a broad window the transparancy of the window might not be uniform over its area.

It seemed desirable to divorce the design of the double spectrometer completely from the single spectrometer and produce an instrument suited to the requirements of a large group of problems likely to be attacked without aspiring to achieve one so universal in its applicability that it would perform none of its functions well.

\section{Design of the Spectrometer}

Fig. 5 is a general view of the spectrometer. Two circular tables $A$ turning on conical pivots with accurately parallel axes support the crystals. The interpivotal distance is 5 inches. The pivot holes are bored in a single bed block of steel $B$, situated under the front end of the instrument. Two flat steel levers extending from the pivots to a similar steel bed block, $C$, under the rear end of the instrument can slide laterally, opening and closing like scissors, on top of this block so as to give equal and opposite rotations to the crystal tables. The levers are given their motion by a transverse shaft $D$, with right and left hand threads passing through nuts pivoted to the ends of the levers. The shaft is prevented from translating axially by a thrust bearing $E$ half way between the levers. This bearing which has no axial play at all has sufficient lateral play to permit the screw shaft to translate in the direction of the crystal pivots as the levers spread apart. The action of the entire system is such that the crystal pivots and the driving nuts at the opposite ends of the two levers always stand at the four corners of a perfectly symmetrical isosceles trapezoid whose base is the screw shaft, whose sides are 
the levers, and whose top is the line of centers of the crystal pivots. The top and sides are of constant length but the base and hence the altitude varies. It is for this reason that the thrust bearing on the screw provides for a slight lateral translation of the screw shaft. This same screw shaft carries a brass drum $F$ on one projecting end on which the scale of glancing angles is engraved. The smallest divisions on this drum represent ten seconds of arc for the glancing $\mathrm{x}$-ray reflection angles, and are separated by about three $\mathrm{mm}$. A very important part of the design is a helical spring $G$ running between two short posts on the under side of the two levers. The tension of this spring holds the levers together thus completely removing all backlash in the screw, nuts and pivots. The crystal tables are provided with angle scales engraved on their peripheries to facilitate setting. The levers can be clamped to and

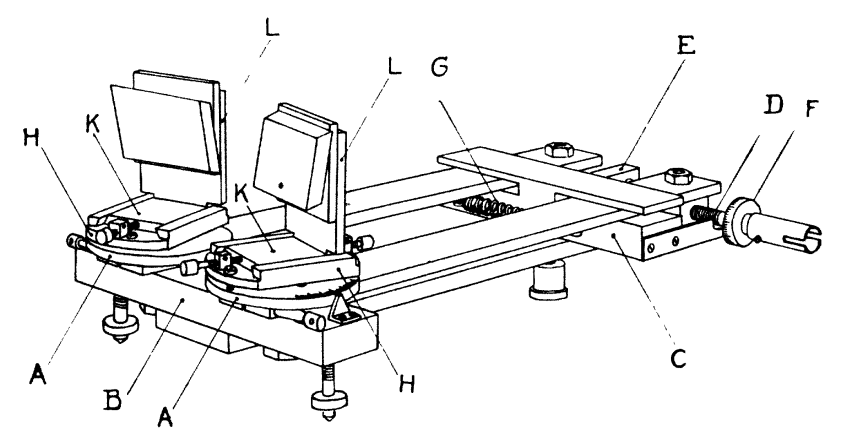

Fig. 5. Double spectrometer providing equal rotation of both crystals with respect to the line of pivot centers.

unclamped from the crystal tables in the same way as the tangent screws on a spectrometer or engineer's transit. The crystal tables are provided with removable false tops $H$ which can rock about a horizontal axis. This is accomplished by two steel ball bearings lying between the crystal table disk and the false top in slight conical depressions provided for them and two opposing screws which clamp the false top to the crystal table with any desired slight inclination to the level. This adjustment is provided to permit of making the crystal faces accurately parallel to the axes of the pivots during the initial optical adjustment. A carriage $K$ bearing the vertical crystal support slides in ways provided on each false top. This adjustment permits the crystal face to be brought in coincidence with the axis of rotation during the initial adjustment. The crystals are each fastened to a brass plate at three points with a very small quantity of beeswax. The brass plate can be fastened to the vertical crystal support $L$ with two screws passing through holes large enough to permit of some adjustment in the position of the crystals. The vertical crystal support has a large hole bored through it at the point corresponding to the center of the crystal face. The reason for this hole is to permit sighting with a telescope and Gauss eyepiece at either side of a small interferometer plate which takes the place of the crystal during some of the initial adjustments. The entire spectrometer stands on leveling screws. 
This instrument was very inexpensive to construct. The frame was built of pieces of cold rolled steel of rectangular section. No hand scraped surfaces were found necessary nor any precise circular divided scales. The one accurate requirement was that the pivot holes in the bed block $B$ should have their axes as nearly parallel as possible. The spectrometer was built by one man in about five and one half days but in spite of its cheapness and simple construction a large amount of surprisingly precise work can be done with it.

\section{The Subsidiary Rotating Mechanism}

As before stated the quantities measured with the double spectrometer are: first, the angle between the crystals ${ }^{9}$ and second, the intensity of the twice reflected $x$-rays. The spectrometer just described measures the first quantity with high precision and reproducibility. It is however an important refinement to provide motion of the ionization chamber and $\mathrm{x}$-ray source relative to the spectrometer in order to avoid the errors caused by shifting of the beam across the crystal faces and kindred objections already mentioned. It should be understood however that the requirement of precision in the motion of source and ion chamber is not as exacting as it is in the angular motion of one crystal relative to the other since the precise wave-length selected by the double reflection does not depend on the position of source or ion chamber so long as these are so situated that some radiation can suffer the double reflection. The wave-length selected by the double reflection depends wholly and solely on the angle between the crystals and it is this quantity which must be and is measured precisely and reproducibly on the drum of the spectrometer.

The motion of $x$-ray source and ion chamber relative to the spectrometer in the instrument here described is accomplished in such a way that the ion chamber remains stationary relative to the room. This means that relative to the room the spectrometer rotates about a center in line with the axis of rotation of the second crystal and at twice the rate of the second crystal. At the same time relative to the spectrometer the $\mathrm{x}$-ray source rotates about a center in line with the axis of rotation of the first crystal at twice its rate of rotation. The scheme of holding the ion chamber stationary was adopted in order to permit the use of a short unarticulated vacuum sleeve surrounding a short lead wire of low and perfectly constant capacity connecting the ion collector to the electrometer. The ion chamber could probably be permitted to move relative to the room by using a vacuum tube amplifier such as has recently been developed for The General Electric Company in a shielded housing attached to the ion chamber to replace the electrometer. There is some question as to whether the enormous sensitivity, stability and reproducibility attainable with the Hoffmann type vacuum electrometer can be matched with the amplifier tube, and work is now being started to investigate the possibilities of this method in connection with a vacuum double crystal spectrometer now in process of design at this Institute.

- The angles for which the drum is calculated are half the supplement of the dihedral angle between the crystals to facilitate their direct interpretation as glancing angles. 
The above mentioned relative motions are obtained by means of two rotating false table tops of ply wood best shown in Figs. 6, 7. Ply wood was used instead of metal because the presence of the $\mathrm{x}$-ray tube fed with high voltage made an insulating material convenient. The ply wood has proved to be quite free from warping and entirely satisfactory. The upper table top, $F$,

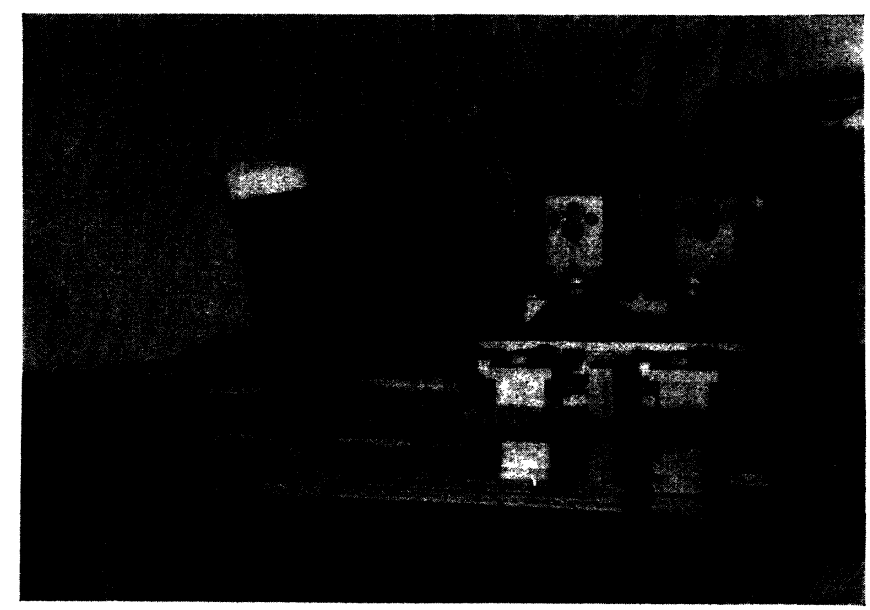

Fig. 6. General view of spectrometer, rotating table tops, rotating mechanism, and x-ray tube housing.

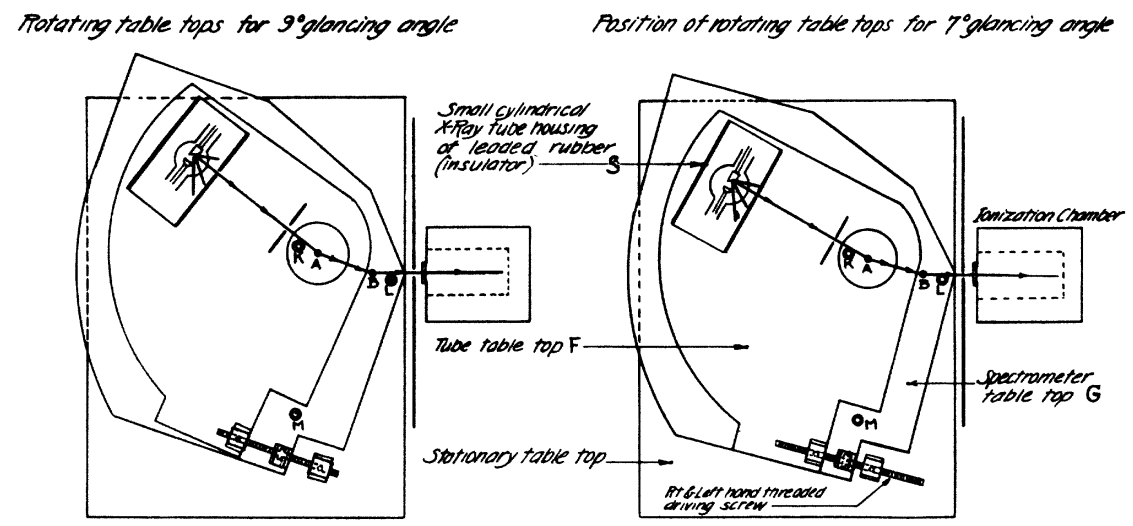

Fig. 7. Schematic diagram of table tops.

Figs. 6 and 7, carries the source of x-rays $S$. It rotates on a pivot centered at $A$ consisting of a disk of ply wood 5 inches in diameter fixed to the second false table top $G$. This second table top in turn rotates on a stationary pivot centered at $B$ and attached to the stationary table top. The centers $A$ and $B$ are separated by the same distance as the interpivotal distance on the spectrometer. The spectrometer rests entirely on the second table top $G$, the position of its three feet being at $K, L$ and $M$. (The reason for the large diameter 
pivot $A$ is here obvious.) The pivots of the crystals are directly above and in line with the table top pivots $A$ and $B$. The table tops are given the required angular rate of rotation by a second right and left hand screw with nuts and thrust bearing almost identical with the one on the spectrometer proper and having the same thread pitch. The two screws are connected through a simple type of universal joint transmission to gears having a ratio of two to one. The post supporting the gears is fastened to table top $G$ and hence is stationary relative to the spectrometer. The entire mechanism is rotated by turning a shaft set at right angles to the screw shafts for accessibility the former driving the latter through bevel gears. This shaft terminates in a hollow brass tube $T$ slotted axially on two diametrically opposite sides. A brass ball on the end of a long insulating handle can be inserted in this tube, and a rotary motion given to the tube by means of a diametral steel pin through the brass ball which engages in the slots of the tube. (The universal joints mentioned above are all of this simple ball and pin and slotted cylinder type and are very satisfactory.)

The kinematics of the table top drive is identical to that of the spectrometer. It is most easily grasped by taking as a reference, not the stationary table, but the false top $G$ on which the spectrometer stands. The thrust bearing is attached to this top, and on turning the screw one nut causes the $\mathrm{x}$-ray source on table $F$ to rotate around the center $A$, relative to the spectrometer standing on $G$, at twice the rate of the crystal at $A$, while the other nut causes the table proper, and with it the room and the ion chamber, to rotate relative to the spectrometer standing on $G$ around the center $B$, at twice the rate of the crystal at $B$. The same provision for lateral displacement of the shaft in the thrust bearing is provided for here as on the spectrometer. The whole device is of course open to the objection that it moves the source and ion chamber not accurately at twice the angular rate of the crystals, but rather at such a rate that the sine of one angle is twice the sine of the other angle. This however introduces far too small an error to cause trouble over the small rotations ordinarily required.

The lever arms of the spectrometer have each a range of motion of a little over one degree on each side of their position of mutual parallelism. The tables have each a range of two degrees on each side of their central position. A great deal of work can be done with even so small a range as this but slight modifications would suffice to increase the range. It is our practise to pick some definite angle setting near the midpoint of the range of glancing angles to be studied and then orient the crystals and other parts so that this angle corresponds to the zero position of the drum $F$ (Fig. 5) with the levers in their central position. Details of this operation are described below.

\section{Minor Spectrometer Details}

The tube housing shown in Fig. 6 consists of a micarta tube large enough to contain the $\mathrm{x}$-ray tube covered with a wrapping of two layers of the leaded rubber $1 / 16$ " thick such as is used by Röntgenologists for protective aprons. This material which can be obtained from surgical supply houses has the 
advantage of being opaque to $\mathrm{x}$-rays, flexible, and a good insulator. Its uses are manifest to anyone who has worked in $\mathrm{x}$-rays. Since it is an insulator one can construct small and therefore light boxes of it to contain $\mathrm{x}$-ray tubes.

Fig. 6 was taken with a copper target tube in the lead rubber housing provided with a projecting goose-neck tube with a charcoal trap dipping into a liquid air container. The latter is plainly visable standing on table $F$. An investigation over a period of two months ${ }^{10}$ was made with this copper target tube which had a mica window sealed on one side with DeKhotinsky cement. The liquid air and charcoal were found quite sufficient to maintain an excellent $\mathrm{x}$-ray vacuum throughout the entire period.

The rotation required of the $\mathrm{x}$-ray tube does not prevent the use of mercury pumps if necessary. These are installed under the table and are hung from table top $F$ through holes bored in $G$ and in the table top proper sufficiently large to permit the complete latitude of rotation. The pumps can be connected directly to the tube with an all-glass connection since no relative motion occurs between the two.

In order to insure smooth and easy operation of the table tops the bulk of the weight of the $\mathrm{x}$-ray tube, housing, pumps and spectrometer is relieved by a simple system of lifting ropes going up to levers and counter weights hanging from the ceiling.

To give greater accessibility the entire table bearing all the parts so far described can be unclamped and moved away from the concrete wall shelf on which the detecting system consisting of electrometer and ion chamber stands.

\section{Design of the Intensity Measuring System}

The essential requirements are that the $\mathrm{x}$-ray intensity measuring system shall have high sensitivity coupled with great stability and reproducibility. It is also convenient to have as large an $\mathrm{x}$-ray window in the ion chamber as possible to make the task of alignment less arduous. Fig. 8 shows a cross section of the entire ion chamber-electrometer system. The chamber is a large cylindrical brass can $A$ nickel plated inside and out. A disk shaped cover $B$ fastens on to the end of the can with six screws, but is electrically insulated from the can with a gasket and insulating washers under the screws. The cover is made gas tight by pressing a fillet of red universal laboratory wax into the groove $C$ provided for it. The $1^{\prime \prime} \times 3^{\prime \prime} \mathrm{x}$-ray window in this cover is covered with a piece of paraffin impregnated Balsa wood-an extremely light yet stiff wood used in airplane model construction-with the grain running across the short dimension of the opening. The wood is attached to the cover with beeswax. The inner surface of the wood is coated with aluminum beryllium alloy 0.0002 inches thick making contact with the metal cover. The purpose of this coating is completely to define the potential all over the window so as to insure that the ions formed in this vicinity will not fail to be collected and also to prevent electrometer disturbances due to charges on the window inducing fluctuating charges on the collector.

10 Dumond and Hoyt, Phys. Rev. 36, 799-809 (1930). 
A grid $D$ of No. 36 nickel wire wound on and spot welded to a light nickel wire frame extends back from the window and surrounds the collector. This grid is supported on the brass cover $B$ and by means of an external wire connected to the cover is brought to the potential of about 135 volts above ground, furnished by radio $B$ batteries. The rest of the can which stands in contact with the electrometer housing is grounded.

The collector is led out of the can through a quartz insulator and as the can is itself grounded no guard ring is necessary. The collector of nickel wire can be fastened with a set screw into a brass cap on the top of the quartz

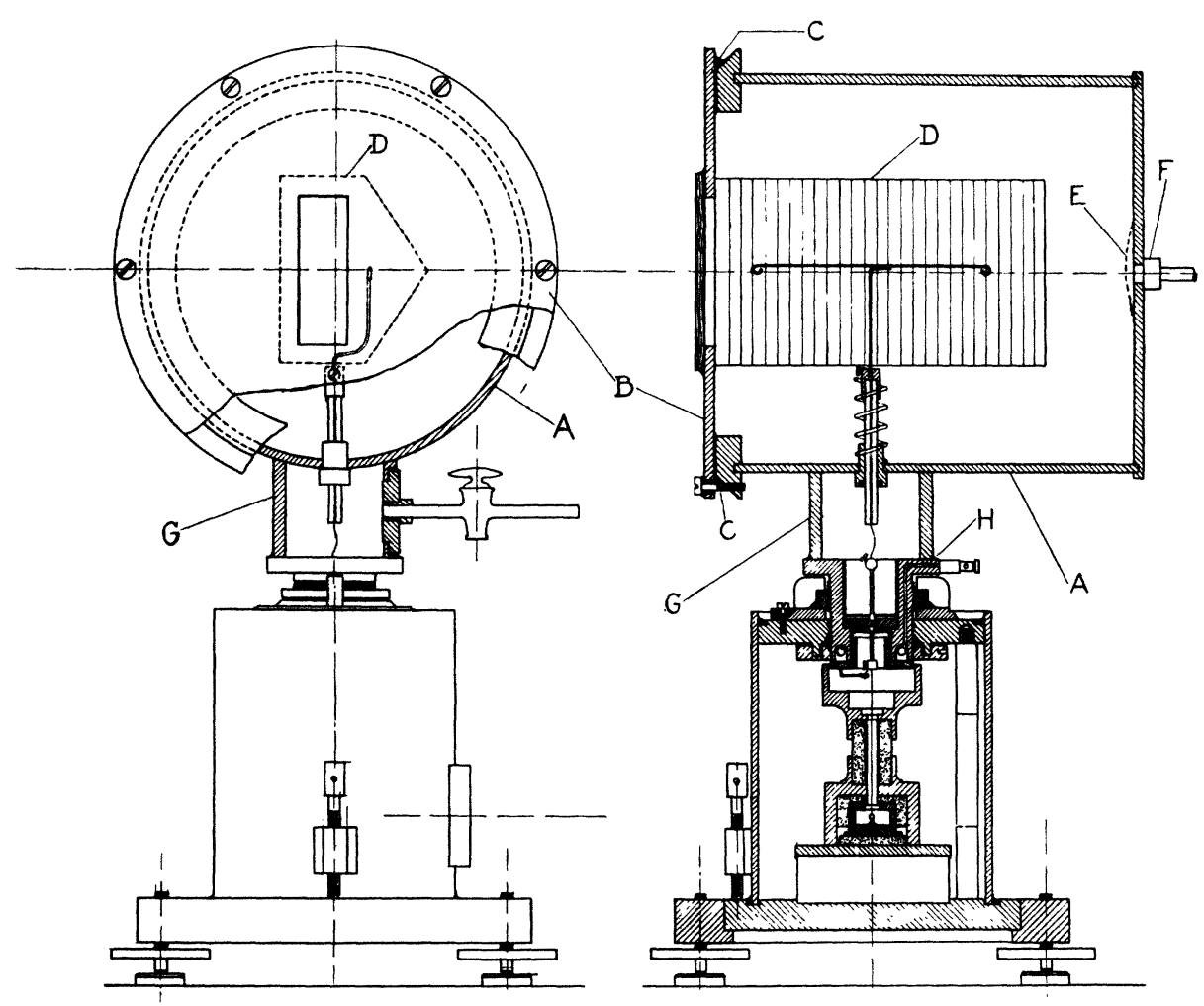

Fig. 8. Complete cross section of x-ray detecting system.

insulator. This collector is put in place through the $\mathrm{x}$-ray tube window just before sealing on the Balsa wood cover. A small piece of fine mesh nickel screen $E$ is welded over the inside opening of the gas connection $F$. This saves having to shield the glass tubes leading into the ion chamber by the usual method of covering them with tin foil.

The purpose of the internal grid is to minimize the fluctuations in the natural leak of the system caused by alpha-particles from the walls of the ion chamber. The space of about $5 \mathrm{~cm}$ from grid to chamber walls filled with about an atmosphere of methyl bromide accomplishes this very well.

The gas outlet from the ion chamber leads to a mercury manometer on 
which any change in the methyl bromide pressure can be read. There is a glass cock for filling and a drying tube in constant connection with the chamber. It is important to keep the quartz insulator dry.

A fine No. 40 wire passing through the quartz insulator connects the collector to the highly insulated suspension of the Hoffman type electrometer. The connection is made through a short evacuated sleeve $G$ which is made vacuum tight against the top flange of the electrometer with a fillet $H$ of red universal wax.

Save for the flat flange just mentioned and for the use of universal wax instead of rubber gaskets to make the cylindrical case of the electrometer vacuum tight there are no changes in the design of the Hoffmann type vacuum electrometer. This remarkably satisfactory instrument has been completely described by its designer ${ }^{11}$ and will not be given space in this article.

\section{Sensitivities and Reproducibilities Obtained}

The spontaneous fluctuations in readings with this $\mathrm{x}$-ray measuring system correspond to about $5 \times 10^{-16}$ amperes. These fluctuations are the limiting feature at present on the smallest currents readable. They are only due in part to alpha-particles from the walls of the chamber. Their source is being studied and it is hoped that they can still be very greatly reduced. With these fluctuations at the present value currents of $6 \times 10^{-14}$ amperes can be read with a reproducibility of one percent in a single reading of the order of a minute's duration. Currents of $10^{-15}$ amperes can be read with fair accuracy by taking several somewhat longer readings and averaging. We have succeeded in detecting the satellite $K \alpha_{34}$ of copper at only $1.75 \mathrm{KV}$ above its critical exitation voltage with this outfit. This satellite had spectral ordinates protruding above the background only one four hundred and fiftieth as high as the maximum ordinate of $K \alpha_{1}$ its parent line six X.U. away.

The electrometer has a very stable zero that will remain constant within a few millimeters for months. The instrument as now used has a rather stiff suspension. Mr. Julius Pearson of this institute has succeeded recently in producing Wollaston wire suspensions for the Hoffmann electrometer with much longer period than the one now in use. The present rather low volt sensitivity could then be increased to about $10000 \mathrm{~mm}$ per volt. The capacity of the whole insulated system of electrometer and ion chamber is about 12 electrostatic units. We therefore do not believe the possibilities of the above described system are exhausted with the results here reported.

\section{Technique of Operation}

Method of orientating the crystals initially.

The procedure about to be described accomplishes in practice the fulfillment of the following required conditions. (These conditions are not all equally exacting.)

1. The crystal reflecting planes must be parallel to the axes of rotation of the crystal table pivots.

${ }^{21}$ G. Hoffmann, Ann. d. Physik 52, [7] 665-708 (1917). 
2. The reflecting surfaces of each crystal must coincide with the axis of rotation of its pivot.

3. The two crystal reflecting planes must make equal angles with the plane through the axes of the pivots.

4. The dihedral angle between the two crystal planes must be set for some known value corresponding to the "zero" drum position of the screw and lever crystal turning mechanism to facilitate "finding" a spectral line.

The first condition is quite important since the accuracy with which the dihedral angle between the crystal faces can be determined and hence the accuracy to which the wave-length scale is known depends on this. Also if condition No. 1 is not fulfilled the so called "horizontal" plane i.e. the plane normal to the dihedral angle formed by the two crystals may be quite oblique to the pivot axes and the general line of the $\mathrm{x}$-ray apertures causing an unsuspected vertical divergence of the rays from the "horizontal" plane. Requirement No. 1 evidently becomes more rigid due to both of these last named effects as the crystal planes approach parallelism.

The second condition cannot be fulfilled very rigidly since the plane of the face of the crystal is ill defined on account of the unavoidable cleavage steps. Its purpose is to minimize the gliding of the selected beam across the crystal face as the crystal rotates.

The third condition merely insures that the radiation from the first to the second crystal shall pass parallel to the line of centers of the two pivots thus facilitating the initial alignment of the apparatus. It need only be fulfilled to within a few minutes of arc.

The fourth condition is merely a convenience. We have however invariably succeeded by the technique about to be described in locating the crystals so closely that it was not necessary to hunt for a known spectral line over a region much greater than its own observed breadth.

A small optical spectrometer with collimator removed and with a Gauss eyepiece in the telescope is used. Calcite cleavage faces as Allison has noted are poor reflectors of visible light at normal incidence. It is however, possible to obtain a sufficiently intense image of the cross hairs for visibility if one employs a strong but small incandescent light, well shielded from directly striking the eye to illuminate the Gauss eyepiece. A small black disk of paper, say $0.5 \mathrm{~mm}$ in diameter, should be carefully attached with a little shellac to the intersection of the cross hairs. The period from a set of gummed letters such as are used to stick on drawings makes a good disk. This makes a spot large enough to see in the faint reflected image. Considerable patience is required in hunting for the faint image but the telescope and Gauss eyepiece furnish such an accurate method of alignment that it is worth while spending an hour or so, especially as it need not be done often.

A glancing angle $\theta_{0}$ is first chosen as a setting for the crystals when the angle drum is set at zero and the levers and drum are then adjusted to that zero position. The optical spectrometer is then set on a bench in front of the $\mathrm{x}$-ray spectrometer in such a position that in sighting the telescope alternately first at crystal one and then at crystal two the optical telescope must turn 
through approximately the angle $2 \theta_{0}$. The pivot axes of both spectrometers are then rendered accurately vertical with the leveling screws by taking reversed readings with a good level. A pair of light plumb lines of white silk thread are then adjusted so that they define a plane the perpendicular bisector of the plane through the two pivot axes. This can be done very precisely with a trammel which is engaged at one end in the conical center holes on which the crystal pivots were turned and is used as a "feeler" to strike four arcs in space centered each from a pivot and just making contact on either side with the plumb lines. The geometry is the simple Euclidian construction for a perpendicular bisector. The telescope is now adjusted to sight accurately down the line defined by the two plumb lines. The index of the divided circle on the optical spectrometer is then set on zero and clamped and the two spectrometers are rechecked for level and sealed to the bench with shellac or wax. The next step is to render the line of collimation of the telescope accurately normal to the pivot axes of the x-ray spectrometer. (Incidently when this is done the line of collimation of the telescope will be horizontal.) A small plane parallel glass interferometer plate is stuck on with wax over the hole in the vertical brass crystal support and trial reflections are made from both sides of this plate turning the crystal table through $180^{\circ}$ each time and correcting half the error at the plate and half in the level adjustment of the telescope (not the spectrometer of course) until the Gauss cross hairs coincide with their reflected image equally well with the plate facing either way. This condition must be fulfilled for both crystal pivots. The telescope collimation line is now accurately normal to both the $\mathrm{x}$-ray and optical spectrometer pivot axes and its level must not be subsequently disturbed. The plane parallel glass plate is now removed and the calcites ready mounted on their brass plates are attached to the vertical crystal supports with screws. Care should be taken to locate them symmetrically over their axes of rotation at the same height above the turntables. At zero reading on the divided circle of the optical spectrometer the telescope is now normal to the plane through the axes of the $x$-ray spectrometer pivots. Turn the telescope through the angle $\theta_{0}$ to right or left of this point as read on the divided circle of the optical spectrometer and sight at the crystal face. The crystal table is then unclamped and orientated and the false top tilted until the cross hair image normally reflected from the calcite coincides with the cross hairs. The crystal reflecting face is brought into coincidence with its pivot axis by screwing the crystal support carriage diametrically across until a tangential view of the face with the crystal rotated to either side the stationary line of sight shows no displacement. This should be done after rendering the crystals vertical but before finally clamping them to the levers at the correct horizontal orientation defined by the telescope.

The entire procedure requires three or four hours and need only be performed once for each pair of crystals used unless some accident disturbs them. The divided circles on the crystal turn tables should be carefully read and recorded. ${ }^{12}$ This gives a fiducial reference from which the proper

\footnotetext{
${ }^{12}$ A scale capable of being read to within a few minutes of arc is sufficiently accurate.
} 
settings for other glancing angles can be made without having further recourse to the Gauss eyepiece reflection method.

\section{Alignment of other parts}

It is necessary also to align the source of $x$-rays in the proper position with respect to the spectrometer. Furthermore the entire system of source and spectrometer must be aligned with respect to the ion chamber so that the rays will enter its window. We have found that this can all be done very conveniently after the crystals have been aligned by cutting a templet out of Bristol board such as is shown in Fig. (9). The edge segments $A, B$, of this templet are fitted against the crystals and the edges $C, D$, then point respectively in the direction of the source and the ion chamber window when their locations are correct. If the templet is carefully laid out with a precision vernier protractor and accurately cut with a razor blade held against a steel

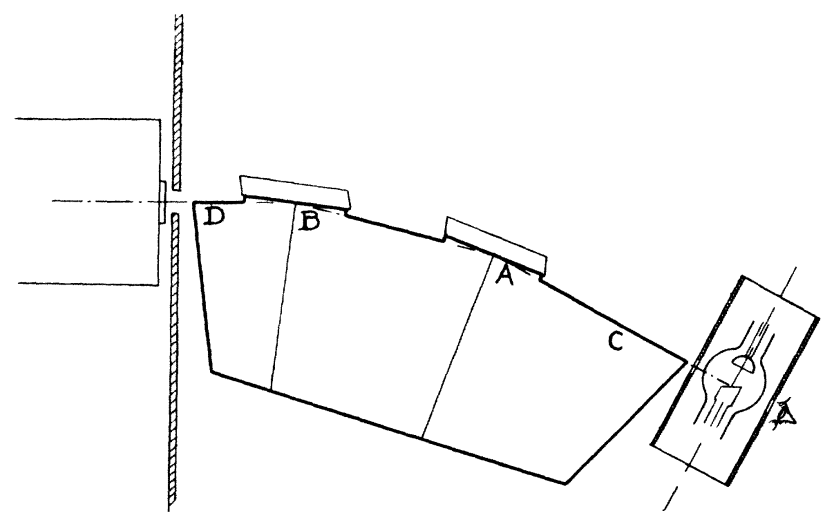

Fig. 9. Cardboard templet used in aligning table tops and x-ray tube.

straight edge the method is accurate to within a few minutes of arc. It is helpful to lay out the normals to the crystal faces on the cardboard. When the cardboard is properly orientated against the crystal faces the reflected image of these normals should line up straight with the normals themselves.

Needless to say the cardboard templet is laid out for the glancing angle $\theta_{0}$ corresponding to the mid value of the spectral range chosen for the particular work to be done and the above described alignment procedure must be carried out with the spectrometer and the ply wood table tops set at the midpoint of their travel, i.e. at the point where the pivots of the nuts on the right and left hand screw shafts are separated by the same distance as the two crystal pivots.

\section{Elimination of BACKGROUND}

The detection and accuracy of measurement of faint lines is limited by the intensity of the $\mathrm{x}$-ray background. Also the shapes and positions of lines are distorted by background structure. It is therefore essential to consider means of reducing background. The causes of background are: 
1. Selective reflection of continuous or "white" radiation from the source.

2. Natural leak of ion chamber and electrometer system.

3. Amorphous or non-selective crystal scattering and fluorescent radiation excited in the crystals.

4. Leakage of $x$-rays without suffering double reflection in the prescribed orders.

It is not always desirable to eliminate all of the above factors. For example if one is interested in studying either the continuous "white" radiation or diffuse lines partaking of the nature of a continuous distribution No. 1 should obviously be retained. Methods of controlling the above sources of background are in the above order as follows.

1. The intensity of white radiation adjacent to a spectral line as measured by the ionization chamber relative to the intensity when the crystals are set for the peak of a line can be very widely controlled by controlling the crossfire or vertical divergence of the $\mathrm{x}$-ray beam. As long as the band of wavelengths selected by the bicrystal reflection is of larger order of magnitude than the natural line breadth, diminution of this band width reduces the background ordinates faster than the line ordinates. The vertical divergence angle is controlled by the height of defining lead openings and their distance apart. In the present instrument one such opening provided with adjustable jaws is arranged in the heavy lead wall dividing the spectrometer and $\mathrm{x}$-ray source assembly from the electrometer and ion chamber.

2. Precautions for minimizing "natural leak" by means of the internal grid in the ion chamber have already been described as have also the use of quartz insulators kept dry with phosphorous pentoxide and the use of vacuum shields and a vacuum electrometer.

3. It is well known that scattering of $x$-rays is of two types namely modified and unmodified scattering. It is also well established that selective crystal reflection is solely of the unmodified type since this is the only type in which the incident and reflected beams are coherent. As regards modified scattering however the two calcite crystals might as well be two pieces of wood or graphite. All wave-lengths of radiation from the x-ray tube can be scattered into the ion chamber by the Compton modified scattering process independent of the angle setting of the crystals. The ratio of modified amorphous scattering to unmodified selective scattering increases with the hardness of the radiation. It is evident therefore that a great advantage in background reduction will be gained by filtering out all radiations from the source harder than those which are in the range being studied. This is especially true when the $x$-ray tube is being operated at voltages considerable higher than the critical voltage necessary to excite the radiation being studied. Thus in the study of the satellite $K \alpha_{34}$ of copper (parent, $K \alpha_{1}$ ) we have found that a filter made of a piece of nickel foil rolled out to $0.0005^{\prime \prime}$ thickness was extremely effective in reducing the ratio of background ordinates to line ordinates.

The same procedure evidently reduces the background due to char- 
acteristic fluorescent radiations excited in the crystals themselves by the harder regions of the continuous spectrum from the x-ray tube.

4. Finally a judicious disposition of lead shields will be found to reduce the background very materially. A vertical lead knife edge should be placed

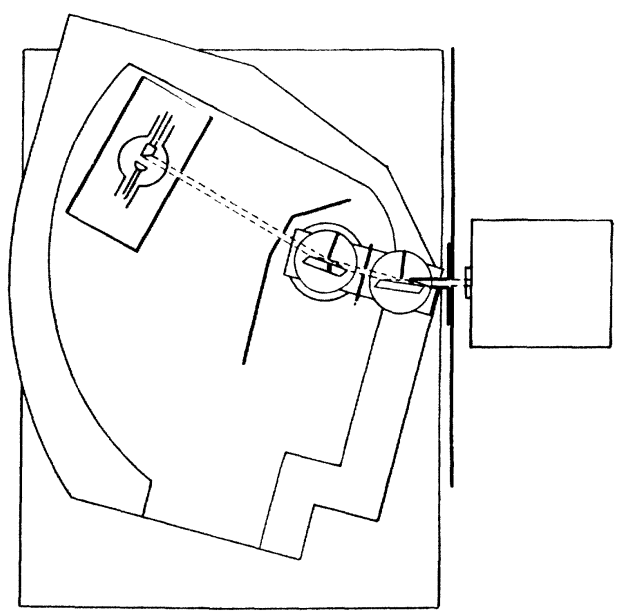

Fig. 10. Scheme of lead shielding.

normal to the reflecting face of each crystal, close enough to the face to prevent any radiation from reaching the ion chamber without suffering the double reflection. These knife edges if placed too close will cut down the

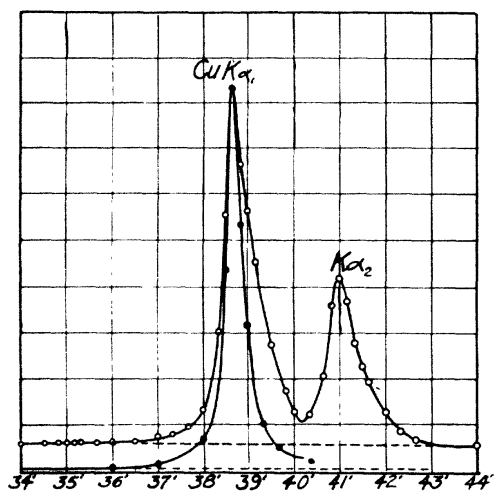

Fig. 11. Comparative curves of $\alpha_{1,2}$ before and after taking the precautions outlined in text for reducing relative background.

available intensity. A little experimentation is the best guide in this matter. A shield with a rectangular window of the proper dimensions placed symmetrically between both crystals is helpful as is also an extensive lead shield near the first crystal between it and the tube. Most effective of all is the use of a lead snout of rectangular cross section which hangs in front of the adjustable lead window in the lead partition between the spectrometer and 
the detecting system and which projects forward to the second crystal. See X Fig. 6. The disposition of this snout if properly chosen will greatly reduce the solid angle from which stray radiation can enter the ionization chamber. Fig. 10 shows the system of lead shields.

The degree to which relative background can be reduced is shown in Fig. 11 by a comparison of the spectral curves of $K \alpha_{1}$ of copper before and

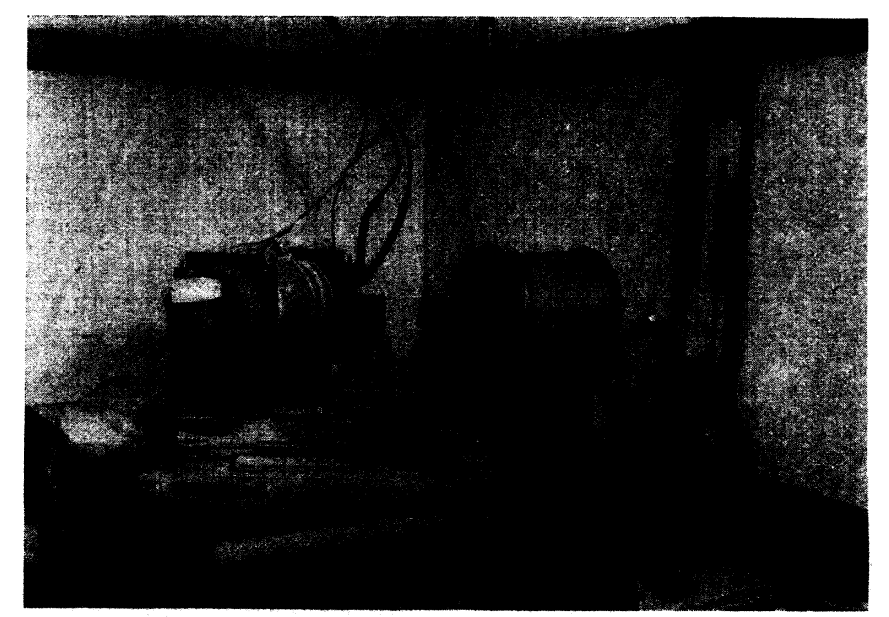

Fig. 12. General view of apparatus with lead shield between spectrometer and ionization chamber removed.

after taking the various precautions discussed above for reducing background. The ordinates of the curve with the low background have been plotted to a scale chosen so as to make both curves coincide at the maximum value of $K \alpha_{1}$. The relative reduction of background is very evident.

Fig. 12 is a general view of the apparatus with the lead shield removed from between ionization chamber and spectrometer. 


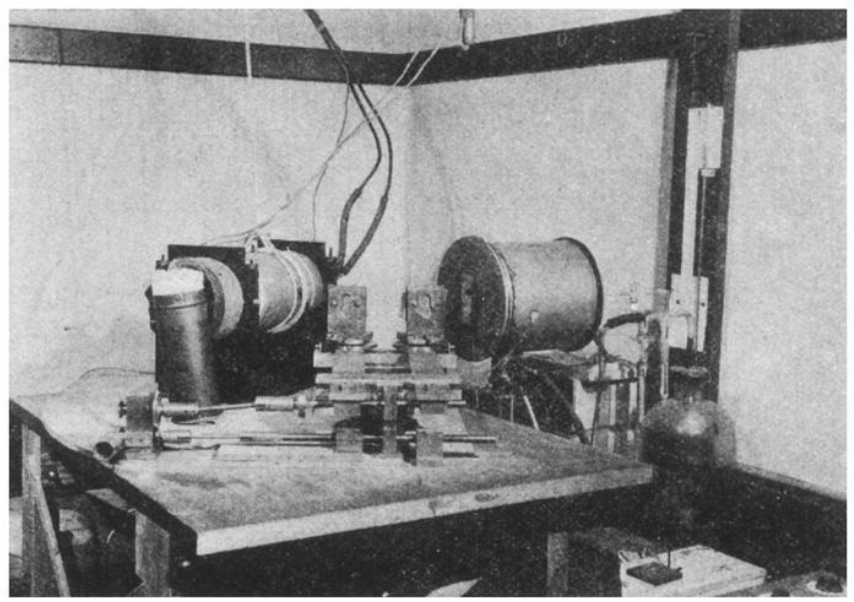

Fig. 12. General view of apparatus with lead shield between spectrometer and ionization chamber removed. 


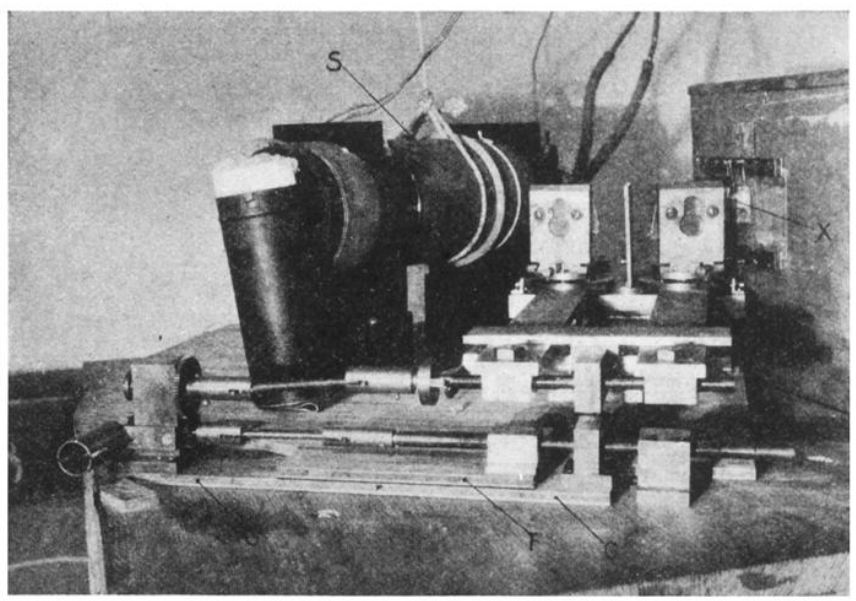

Fig. 6. General view of spectrometer, rotating table tops, rotating mechanism, and x-ray tube housing. 